\title{
Advanced Active Control of Contact Force between Pantograph and Catenary for High-Speed Trains
}

\author{
Yoshitaka YAMASHITA, Dr. Eng. \\ Assistant Senior Researcher, \\ Mitsuru IKEDA \\ Current Collection Laboratory, Railway Dynamics Division
}

\begin{abstract}
This paper presents a method for suppressing the contact force fluctuation between pantograph and catenary for high-speed trains using pantographs equipped with two types of active control technology. One is PID active pantograph control, which utilizes the contact force as a feedback signal to the controller. The other is impedance control, which employs the frame displacement as a feedback signal. Impedance control outperforms PID control in terms of the reduction in contact force fluctuation due to lower noise contamination of the frame displacement signal compared with that of the contact force signal.
\end{abstract}

Keywords: pantograph, contact force, active control, impedance control, current collection

\section{Introduction}

Catenaries provide electric power to railway vehicles through contact with pantographs. The pantograph's raising mechanism supplies upward force to the pantograph head, enabling it to make contact with the catenary. In a resting state, the contact force between the pantograph and the catenary is identical to the force given by the raising mechanism. However, when the pantograph runs along the catenary, contact force fluctuates due to phenomena such as aerodynamic force, propagation and reflection of the wave on the catenaries and changes in the dynamic characteristics of the catenary system depending on position. An excessively large contact force can damage both the pantograph and the catenary or may in the worst case scenario, cause a severe accident like the breaking of the contact wire. On the other hand, if the force exerted is too small, the pantograph and the catenary may easily lose contact, also known as contact loss. Contact loss can cause electrical damage to both the pantograph and the catenary due to arcing or cause disruption to the power supply from the catenary to the vehicle. Contact force fluctuation should therefore be kept as small as possible. Basically, contact force fluctuation of a pantograph at high speed can be kept to a minimum by making use of light-weight materials for pantograph components. Moreover, it is widely known that longer-stroke pantograph head springs ensure better contact performance.

It is also important for pantographs used on Japanese high-speed trains to meet low-noise requirements since noise regulations in Japan are very strict. Noise reduction strategies have been applied by reducing the number of pantograph members exposed to airflow. The reduction in the number of members without losing mechanical strength and current capacity tends to make each of the remaining members larger and/or heavier, thus there is a limit to how much the total weight of the lownoise pantograph can be reduced. In addition, application of long-stroke springs to the low-noise pantograph for the purpose of obtaining high current collection performance actually works against reducing aerodynamic noise because of the longer casing required to cover the springs, which can be another noise source.

As pointed out above, even under ordinary conditions it would be difficult for pantographs to satisfy both high current collection performance and low-noise emission, unexperienced higher speeds making such objectives even harder to achieve. In view of future demand, the authors are working on active control techniques applied to pantographs as one effective method for suppressing contact force fluctuations. This paper presents an active control method for reducing contact force fluctuation between a pantograph and a catenary based on two different control laws.

\section{Reduction of pantograph contact force fluctuations using active control techniques}

\subsection{Active pantograph}

In order to apply an active control technique to the pantograph, a pneumatic cylinder is installed in parallel with the raising mechanism in this study. Pneumatic cylinders generally have a low-speed response compared with other actuators, however greater importance is attached to electrical isolation rather than the response speed. The pantograph equipped with the pneumatic cylinder is shown in Fig.1. This pantograph is utilized on Shinkansen trains and is characterized by its low-noise properties. Figure 2 illustrates the dynamic model of the pantograph shown in Fig.1. Pantographs are often expressed by twoor three-mass dynamic models as shown in Fig. 2 for analytical purposes. $f_{C}$ expresses the contact force. The lumped masses $m_{1}$ and $m_{2}$ correspond to the pantograph head and the articulated frame (general term for upper and lower frames). The raising mechanism gives a constant upward force $P_{0}$ to the pantograph. The spring element $k_{1}$ interconnecting the two masses corresponds to the pantograph head spring. A damping element $c_{1}$ is introduced to take energy dissipation into account. Another damping element $c_{2}$ corresponds to the pantograph damper that is actually installed in parallel with the rais- 
ing mechanism. The actuator is expressed simply as a force $f_{A}$ in this dynamic model. The equation of motion for this model is expressed by the following equations:

$$
\left\{\begin{array}{l}
m_{1} \ddot{x}_{1}+c_{1}\left(\dot{x}_{1}-\dot{x}_{2}\right)+k_{1}\left(x_{1}-x_{2}\right)=-f_{C} \\
m_{2} \ddot{x}_{2}+c_{1}\left(\dot{x}_{2}-\dot{x}_{1}\right)+c_{2} \dot{x}_{2}+k_{1}\left(x_{2}-x_{1}\right)=P_{0}+f_{A}
\end{array}\right.
$$

This actuation method of applying the actuation force to the frame is called "frame actuation." Another method is known as "head actuation," in which the actuation force is applied to the pantograph head directly [1]. The latter method offers the advantage that both the contact force and controlling force generated by the actuator are acting on the same part of the pantograph. This, however, has the disadvantage of an increased pantograph head weight, which generally leads to the poor pantograph / catenary compliance characteristics. For this reason, the frame actuation method was adopted in this study. Hereinafter, the pneumatic cylinder is referred to simply as the actuator. The pantograph is controlled by means of PID control and impedance control methods, to reduce contact force fluctuation. The controller outputs command voltage based on a control law. Then the servo amplifier generates a current proportional to the voltage in order to drive the actuator. For simplicity, in this study it is assumed that the controlling force is also proportional to the current.

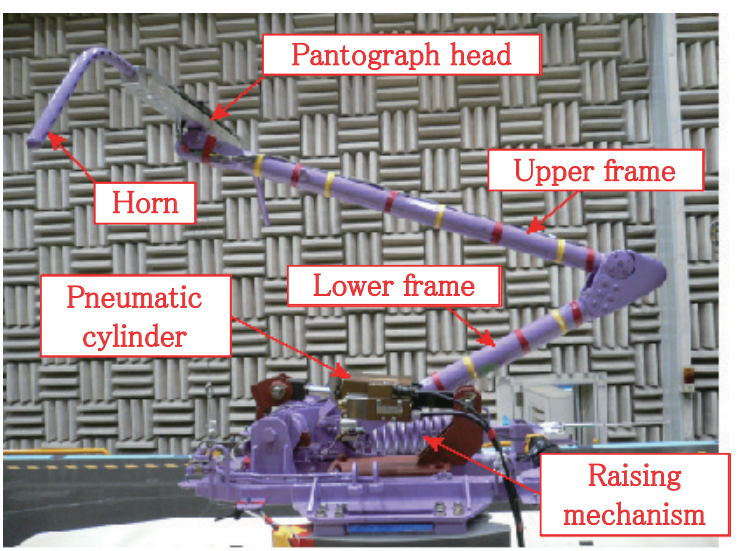

Fig. 1 Active controlled pantograph

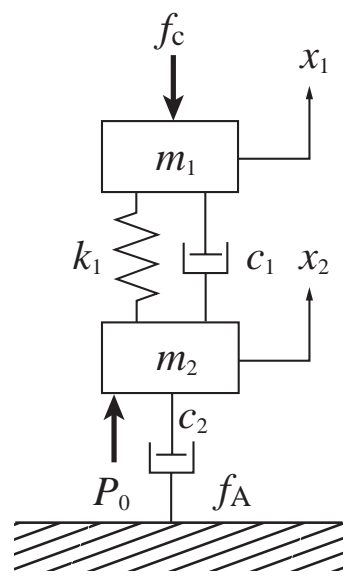

Fig. 2 Dynamic model of the pantograph with pneumatic cylinder

\subsection{PID control}

The pantograph head shown in Fig. 1 is equipped with accelerometers and strain gauges, and is capable of measuring contact force by the taking weighted sum of the sensor outputs. The measured contact force is directly input to the PID controller. The PID controller calculates the error between the measured contact force and the prescribed contact force $P_{S}$, which is equal to the constant upward force $P_{0}$ in this study. In this PID control technique, the controlling force of the PID control is generated based on the following equation:

$$
f_{A}=K_{P} e+K_{I} \int e d t+K_{D} \frac{d e}{d t}
$$

where $e$ is the error calculated by

$$
e=P_{S}-f_{C}
$$

and, $K_{P}, K_{I}$ and $K_{D}$ are proportional, integral and derivative gains, respectively. These gains are all positive values. One finds that if the contact force exceeds the static upward force, the error becomes negative, and the actuator then creates a downward force to cause the pantograph frame to reduce the contact force.

\subsection{Impedance control}

Impedance control is the method by which the controlling of an object is subject to mechanical interaction between itself and the environment; the actuator applies the controlling force to the object until the desired mechanical impedance is created between the object and the environment [2]. Mechanical impedance is defined by a transfer function between velocity of the object and external forces working on it. It is well known that the mechanical impedance between the pantograph and the catenary should be as small as possible in order to obtain the best current collection performance [3][4]. Impedance control techniques are applied to the pantograph to control the mechanical impedance defined by the transfer function between the pantograph head velocity and the contact force. In this study, the pantograph is controlled by impedance control in such a way that its dynamic characteristics are equivalent to those of the model shown in Fig. 3. In this model, a mechanical element called a Maxwell damper, composed of a spring $k_{V}$ and a damper $c_{V}$ connected in series, is introduced. $k_{V}$ and $c_{V}$ are described in this paper as virtual stiffness and virtual damping, respectively. In order to realize this situation, the actuator needs to imitate the Maxwell damper element, i.e. the actuator has to generate a force which is equal to the reactive force exerted by the Maxwell damper element. The equation of motion for the model shown in Fig. 3 can be expressed as follows:

$$
\left\{\begin{array}{l}
m_{1} \ddot{x}_{1}+c_{1}\left(\dot{x}_{1}-\dot{x}_{2}\right)+k_{1}\left(x_{1}-x_{2}\right)=-f_{C} \\
m_{2} \ddot{x}_{2}+c_{1}\left(\dot{x}_{2}-\dot{x}_{1}\right)+c_{2} \dot{x}_{2}+k_{1}\left(x_{2}-x_{1}\right)+k_{V}\left(x_{2}-x_{V}\right)=P_{0} \\
c_{V} \dot{x}_{V}+k_{V}\left(x_{V}-x_{2}\right)=0
\end{array}\right.
$$

where $x_{V}$ is the connecting point of the spring and the damper in the Maxwell damper element. $x_{V}$ is also known as virtual point displacement. $k_{V}$ is determined to ensure that the local minimal point of the mechanical impedance 
curve of the pantograph as a function of frequency, appears at the target frequency $f_{t}$. The relationship between the target frequency $f_{t}$ and the virtual stiffness $k_{V}$ is obtained by an approximated dynamic model of Fig. 3 as follows:

$$
k_{V}=\frac{m_{1} m_{2} \omega_{t}^{4}-\left(m_{1}+m_{2}\right) k_{1} \omega_{t}^{2}}{\left(m_{1} \omega_{t}^{2}-k_{1}\right)}=\frac{m_{2} \omega_{t}^{2}\left(\omega_{t}^{2}-\omega_{0}^{2}\right)}{\left(\omega_{t}^{2}-\omega_{1}^{2}\right)}
$$

where $\omega_{t}=2 \pi f_{t}, \omega_{0}=\sqrt{\left(m_{1}+m_{2}\right) k_{1} /\left(m_{1} m_{2}\right)}$ and $\omega_{1}=\sqrt{k_{1} / m_{1}}$. The frequency $f_{0}=\omega_{0} /(2 \pi)$ is equal to the local minimal frequency of the mechanical impedance curve of the pantograph without the actuator. Figure 4 shows how the mechanical impedance of the pantograph changes by varying $k_{V}$. The horizontal axis represents the frequency normalized by $f_{0}$. The normalized target frequency varies from 0 to 0.8 with 0.2 increments. $k_{V}=0$, which corresponds to $f_{t}=0$ or $f_{t}=f_{0}$, means that no actuation force is applied to the pantograph. From Fig.4, the local minimal frequency appears at $f_{t}$ by varying $k_{V}$ based on the (5), so that it is possible to control the local minimal frequency of the mechanical impedance of this pantograph by changing $k_{V}$. All the parameters, which characterize the dynamics of this pantograph, are listed in Table 1 . These parameters are almost equal to the indentified parameters of the pantograph shown in Fig. $1 . c_{V}$ is set to be $2400 \mathrm{Ns} / \mathrm{m} . c_{V}$ also affects mechanical impedance characteristics, however

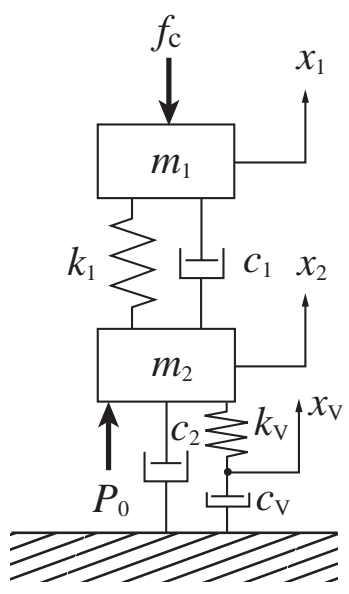

Fig. 3 Dynamic model of the pantograph controlled by impedance control technique

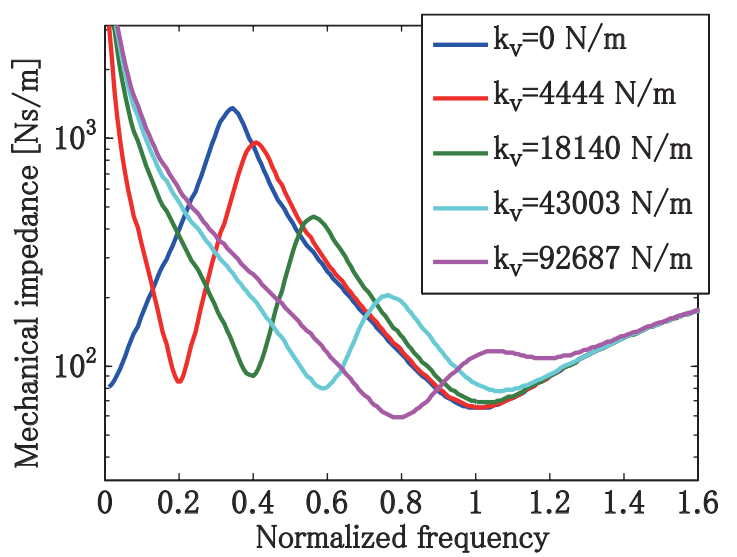

Fig. 4 Change in mechanical impedance of the pantograph to virtual stiffness $k_{V}$
Table 1 Pantograph model parameters

\begin{tabular}{|c|c|c|c|c|c|}
\hline$m_{1}$ & $m_{2}$ & $k_{1}$ & $c_{1}$ & $c_{2}$ & $P_{0}$ \\
\hline $2 \mathrm{~kg}$ & $15 \mathrm{~kg}$ & $11470 \mathrm{~N} / \mathrm{m}$ & $50 \mathrm{Ns} / \mathrm{m}$ & $80 \mathrm{Ns} / \mathrm{m}$ & $54 \mathrm{~N}$ \\
\hline
\end{tabular}

detailed investigation into this would have to be done in the context of future work. On comparison of (4) with (1), the force that the actuator should generate to imitate the Maxwell damper element is expressed as follows:

$$
f_{A}=k_{V}\left(x_{V}-x_{2}\right)
$$

From the equation of motion (4), the virtual point displacement $x_{V}$ can be calculated from

$$
\dot{x}_{V}=\frac{k_{V}}{c_{V}}\left(x_{2}-x_{V}\right)
$$

by the impedance controller. One can find that the only necessary information to apply the impedance control technique to the pantograph is frame displacement $x_{2}$.

\section{Vibration tests}

\subsection{Outline}

In order to investigate the control performance of the active pantograph, vibration tests were conducted. The pantograph was excited at its head by a vibration exciter. The exciter harmonically moves only in the vertical direction. A load cell was installed between the pantograph head and the exciter to measure the true value of the contact force. The exciter and the load cell were bolted, and the pantograph head and the load cell were bonded so that the pantograph basically always remained in contact with the exciter unless an extremely large downward force was applied to the pantograph. The contact force fluctuations of the actively controlled (active) and uncontrolled (passive) pantograph were compared to evaluate the control performance.

\subsection{PID control}

When the pantograph was controlled by PID control technique, the contact force was measured and input to the controller. As mentioned in the last chapter, the contact force could be measured by sensors embedded in the pantograph head; however the contact force measured by the load cell placed between the pantograph head and the exciter was utilized in the tests. Figure 5 shows the block diagram of the pantograph with the PID controller. For convenience, the command signal to the actuator in this diagram is expressed in units of force. Figures 6 show the time series data for pantograph head displacements (excitation displacements), contact forces and command voltages for the active and passive pantographs. The pantograph head was excited by the vibration exciter whose displacement amplitude was set to produce a contact force fluctuation amplitude of the passive pantograph of about $20 \mathrm{~N}$. The PID control gains were set to be $K_{P}=7.5, K_{1}$ and $K_{D}=0$ (P control). The excitation frequency was $0.5 \mathrm{~Hz}$ for Fig.6(a), and $1.0 \mathrm{~Hz}$ for Fig.6(b). One could find that the contact force fluctuation was suppressed by the PID con- 
trol method when the excitation frequency was $0.5 \mathrm{~Hz}$. However, when the excitation frequency was increased to $1.0 \mathrm{~Hz}$, it was clear that the reduction in contact force fluctuation was insignificant. The blue line in Fig. 7 indicates the peak to peak value of the contact force of the PID control based active pantograph as a function of excitation frequency. This value is normalized by that of the passive pantograph. The results plotted as the red line will be discussed later. When the excitation frequency was $1.5 \mathrm{~Hz}$ or more, contact force fluctuation was not suppressed by the PID control. This means that this control method was effective only for slow pantograph head motion. The frequency components in the contact force signal which were above the fundamental (excitation) frequency grew and became more dominant as the excitation frequency increased because of the nonlinearity inherent in the pantograph. The actuator force however was not able to keep pace with such a quick change in the command signal because of the phase delay in the actuator. In general, pneumatic actuators have low response speeds, and the phase delay between the actuator force and the command signal grows as the command signal changes become faster. If the excitation frequency was increased to $3.5 \mathrm{~Hz}$ with the same control gains, the higher frequency component in the contact force signal would eventually make the control system unstable. A positive derivative gain $\left(K_{D}>0\right)$ is usually applied with the hope of improving the response speed of the actuator. However, in this case it amplified the higher frequency component signal and simply made the system more unstable. Application of a low pass filter to the feedback signal seemed to be a promising way to improve the control performance, but it was not easy to design such a low pass filter that
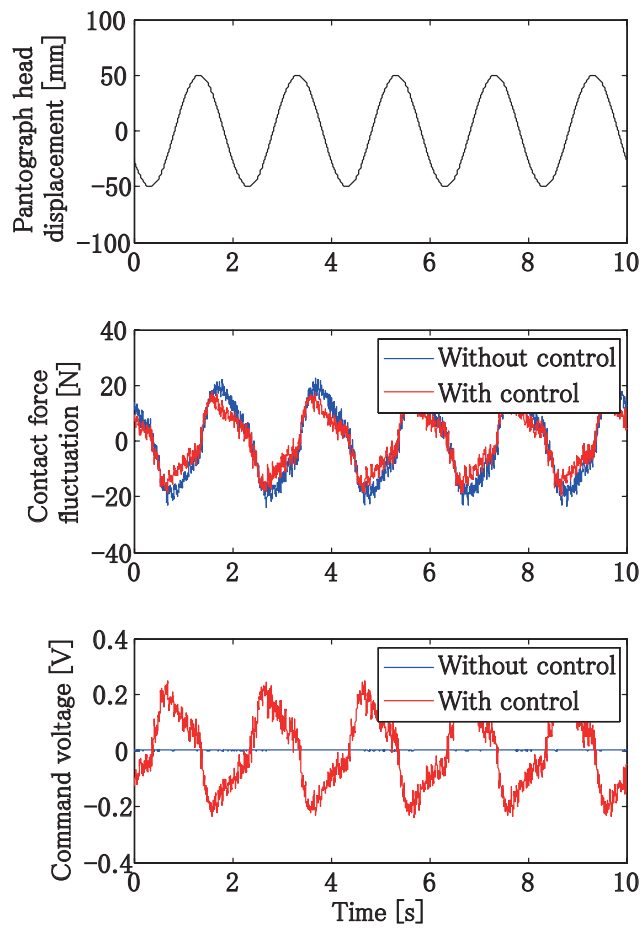

(a) Excitation frequency: $0.5 \mathrm{~Hz}$

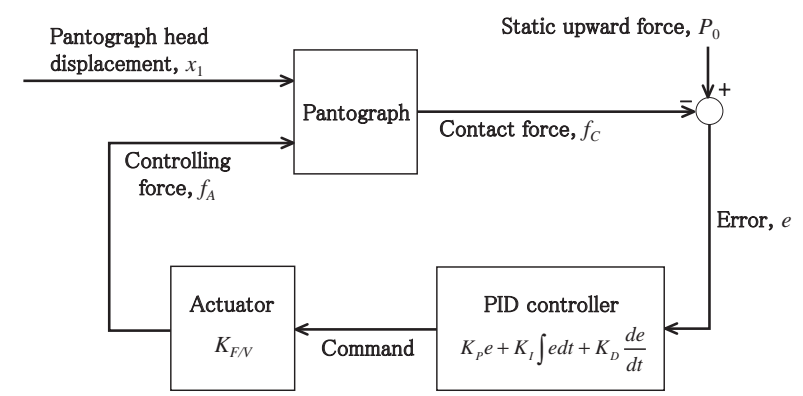

Fig. 5 Block diagram of pantograph with PID controller

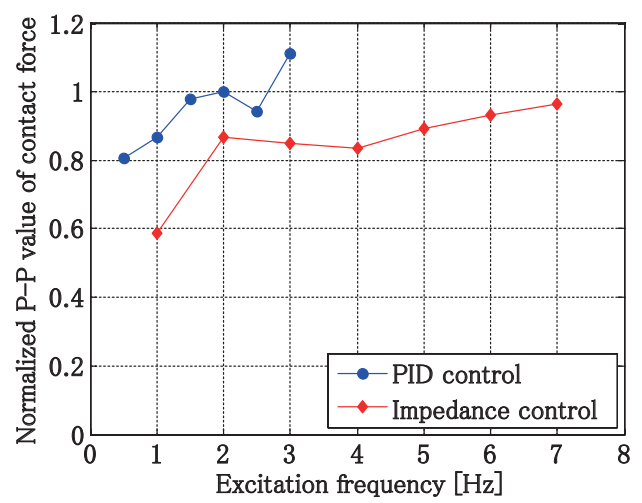

Fig. 7 Statistical values of contact force of the pantograph with and without PID control

could cut off the unnecessary frequency range and prevent the increasing phase delay between the command signal and the controlling force because the required and undesired frequency ranges were relatively close to each
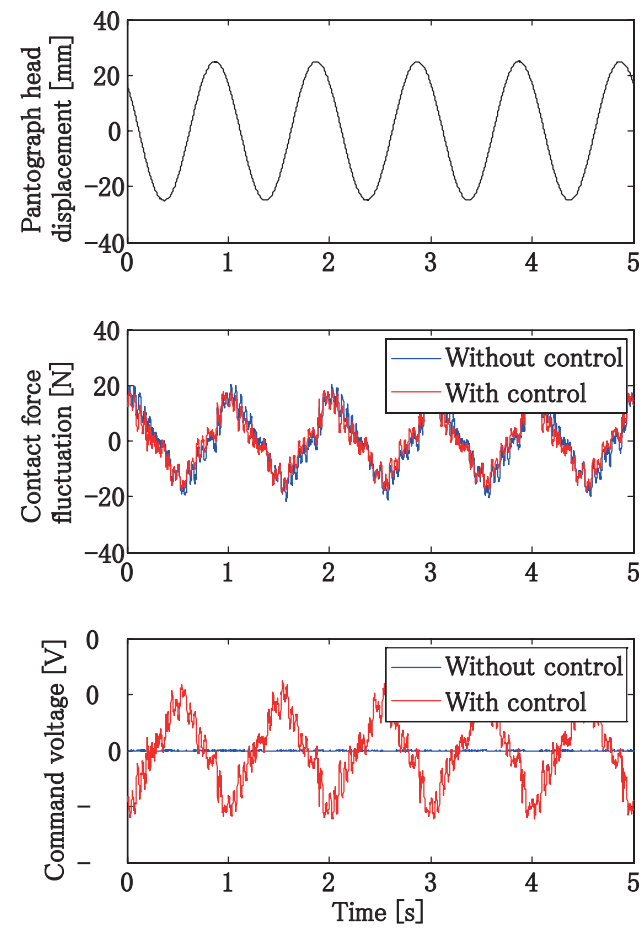

(b) Excitation frequency: $1.0 \mathrm{~Hz}$

Fig. 6 Measured data in the vibration tests for the pantograph with and without contact force feedback based PID controller 
other. From the aforementioned reasons and Fig.7, it is possible to conclude that with respect to the PID based active pantograph, it is possible to suppress the contact force fluctuation for frequencies up to $1.0 \mathrm{~Hz}$.

\subsection{Impedance control}

Under the impedance control technique, the pantograph frame displacement is used as the feedback signal to the controller as stated previously. A contactless laser displacement sensor was used to measure the pantograph frame displacement in this study. Figure 8 shows the block diagram of the impedance control based active pantograph. The command signal in this figure is also expressed in units of force as with Fig.6. Figure 9 shows the time series data for pantograph head displacements (excitation displacements), contact forces, command voltages and frame displacement for the active and passive pantographs. The excitation amplitude was set as mentioned in the PID control case. In this control method, the target frequency $f_{t}$ for calculation of the virtual stiffness $k_{V}$ was varied with the excitation frequency as described in section 2.3. Analytically, the best performance in terms of reduction of contact force fluctuation can be observed when the target frequency is consistent with the excitation frequency. The experimental results obtained in this study however show that the best performance can be observed when the target frequency is slightly deviated from the excitation frequency. This is because of a model-
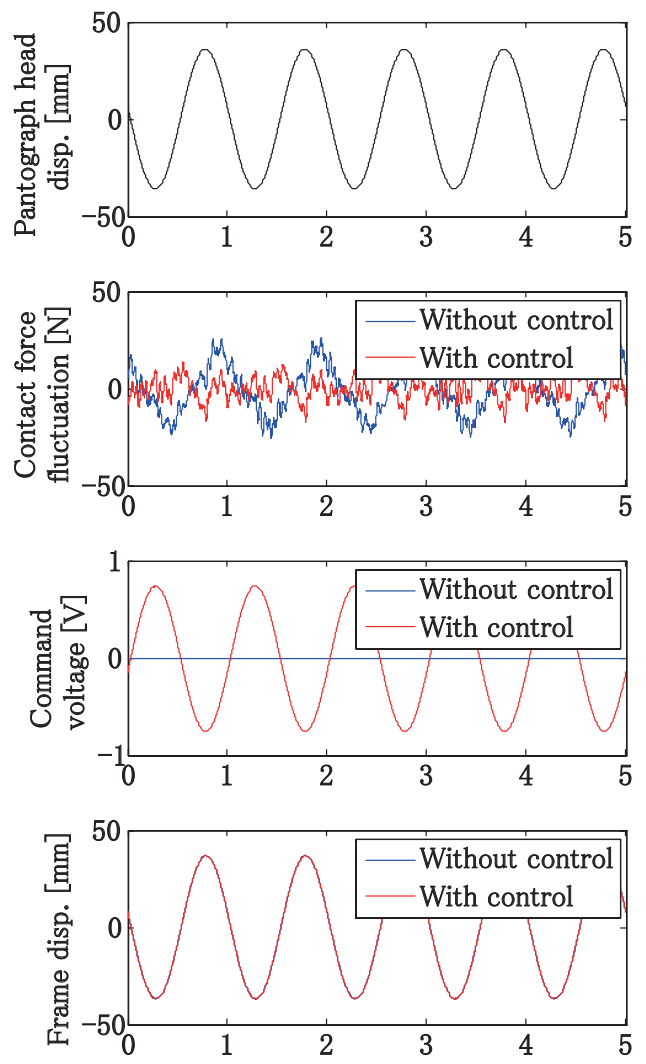

(a) Excitation frequency: $1.0 \mathrm{~Hz}$

$$
f_{t}=0.9[\mathrm{~Hz}]
$$

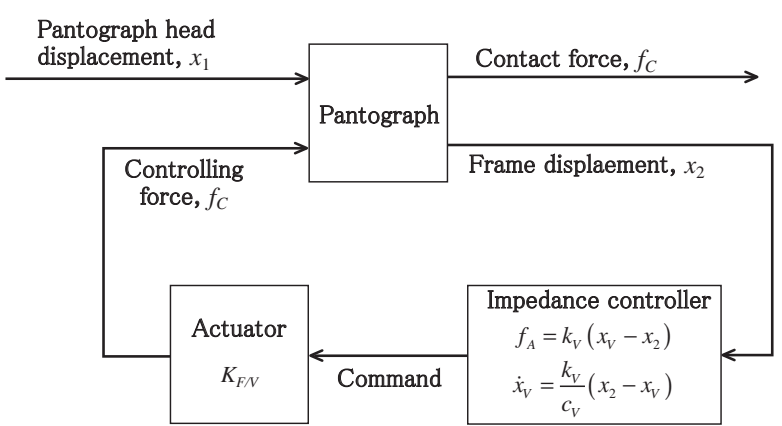

Fig. 8 Block diagram of pantograph with impedance controller

ing error, i.e. the error in identifying the parameters listed in Table 1, was more or less unpreventable, causing possible unmodeled dynamics such as nonlinearity of the actuator or the pantograph. This paper presents the results obtained by the most effective $f_{t}$ for each excitation frequency. Virtual damping was also set to be $c_{V}=2400$ $\mathrm{Ns} / \mathrm{m}$ as mentioned in section 2.3. The excitation frequency is $1.0 \mathrm{~Hz}$ for Fig.9(a), and $4.0 \mathrm{~Hz}$ for Fig.9(b). The red line in Fig. 7 indicates the normalized peak to peak values of the contact force as functions of the excitation frequency. Judging from Fig. 7, the impedance control was effective in the frequency range up to $7.0 \mathrm{~Hz}$. When the excitation frequency was increased to $8.0 \mathrm{~Hz}$, the contact force fluctuation of the impedance control based panto-
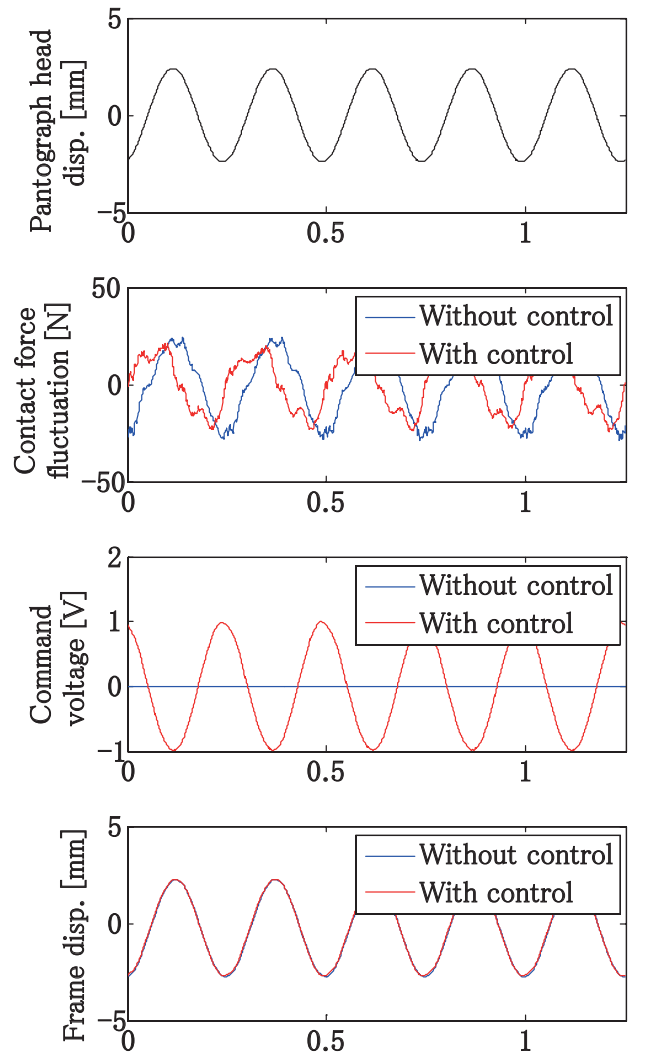

(b) Excitation frequency: $4.0 \mathrm{~Hz}$

$$
f_{t}=4.0[\mathrm{~Hz}]
$$

Fig. 9 Measured data in the vibration tests for the pantograph with and without impedance controller 
graph became greater than that of the passive pantograph. However, even in the high frequency region such as from $8.0 \mathrm{~Hz}$ to $10.0 \mathrm{~Hz}$, the system did not become unstable. From these results, it is obvious that impedance control is more effective and stable than PID control. This is because the pantograph frame displacement signal, which is used as the feedback signal in this control method, contained less noise compared with the contact force signal, and the actuator was able to generate the force following the command voltage signal without making the control system unstable.

\section{Summary}

This paper introduces a method for controlling pantographs to suppress contact force fluctuations using two different control techniques. One was a PID control technique, and the other was an impedance control technique. With respect to the PID based active pantograph, it was able to suppress the contact force fluctuation for frequencies under about $1.0 \mathrm{~Hz}$. The performance limitation in relation to frequency was basically attributed to the lowspeed response of the actuator and also utilization of a contact force signal, which is containing higher frequency components, as a feedback signal. Application of a low-pass filter or a derivative controller did not help to improve the performance of this PID control based active pantograph. The former actually magnified phase delays between the input and the output of the actuator. The latter amplified the higher frequency components of the feedback signal and made the control system unstable. On the other hand, the impedance control based active pantograph, demonstrated the ability to reduce contact force fluctuations for frequencies up to $7.0 \mathrm{~Hz}$. From these results, it is possible to conclude that the impedance control based active pantograph shows better performance than the PID controlled pantograph for suppressing contact force fluctuation.
Other work [5] done by the authors showed that the control performance of the active pantograph significantly worsened when the controlling force was equivalent to or less than the frictional force existing in the actuator. The amplitude of the pantograph head excitation in this study was greater than that in other work, so that performance limitations due to friction were not observed.

The impedance control based active pantograph introduced in this study does not have the ability to control the DC component contact force in principle because setting the target frequency to zero prevents actuation force generation. The PID control however can adjust the DC component by using an integral controller. The two control methods introduced in this paper are therefore complementary, and combining the two control methods could lead to an another powerful control method would be investigated in the future.

\section{References}

[1] G. Diana, A. Collina, F. Fossati, and F. Resta, "Active Control of High Speed Train Pantographs," Third International Conference on Motion and Vibration Control, (1996-9), pp.110-114.

[2] Neville Hogan, "Impedance Control: An Approach to Manipulation: Part I-III," Journal of Dynamic Systems, Measurement and Control, Vol.107, pp.1-24, 1985.

[3] Mitsuo Aboshi, "Influence of dropper spacing of compound-catenary on contact force fluctuation," Proceedings of Jrail2004, pp.117-120, 2004.

[4] Mitsuo Aboshi, "Influence of Pantograph Compliance Characteristics on Contact Force Fluctuation," RTRI report, Vol.18, No.8, pp.17-22, 2004 (in Japanese).

[5] Yoshitaka Yamashita and Mitsuru Ikeda, "Advanced Active Control of a Contact Force between a Pantograph and a Catenary for a High-speed Train," Proceedings of WCRR2011 (CD-ROM), 2011. 